\title{
Online Monitoring the Water Contaminations with Optical Biosensor ${ }^{\dagger}$
}

\author{
Hui Gao *, Silvia Generelli and Friedrich Heitger \\ Centre Suisse d'Electronique et de Microtechnique (CSEM), Center Landquart CH-7302, Switzerland; \\ silvia.generelli@csem.ch (S.G.); fheitger@swissonline.ch (F.H.) \\ * Correspondence: hui.chai-gao@csem.ch; Tel.: +41-81-307-8106 \\ + Presented at the Eurosensors 2017 Conference, Paris, France, 3-6 September 2017.
}

Published: 8 September 2017

\begin{abstract}
This work presents a demonstrator for online monitoring of pesticides or water contaminants which is robust, fast, specific and low cost. The system is designed for continuous monitoring in water matrices (source- and surface water, treated waste water), and is thus completed with an automated fluidics. The main characteristics of the system are: (a) Detection based on competitive immunoassay; (b) Label-free sensing with possibility of following the binding kinetics of the contaminants; (c) Modular sensing surfaces characteristics; (d) In-assay calibration/normalization; (e) Re-generable biochip > 80 times; (f) Detection limit of the analyte, the pesticide atrazine, $0.05 \mu \mathrm{g} / \mathrm{L}$; (g) Computational Bioanalytics; (h) Compensation of the interfering effects.
\end{abstract}

Keywords: label-free detection; real-time online monitoring; competitive immunoassay; water contaminants; pesticides; re-generable biochip; auto-fluidics

\section{Introduction}

There are several well established methods to detect and quantitate contaminants (e.g., pesticides) in drinking and source water by bioanalytical means. Most of them require a laboratory environment with a dedicated and usually bulky instrumentation as well as specifically trained personnel. The target of this work is the development of a bioassay platform with the following characteristics: (i) Reliable detection of several water contaminants, with sensitivities within the regulatory scope, and a time to result of less than $1 \mathrm{~h}$. (ii) User-friendly, featuring a high degree of automation and operation autonomy combined with robustness of operation. The detection platform has to be operated by personnel not specially trained in executing bioassays. (iii) Cost efficiency concerning instrumentation as well as consumables.

It represents quite a challenge to fulfill all these requirements in one and the same bioanlytical platform as immuno-reactions, which are mostly the basis for biosensing devices, are inherently decisive and prone to variation. The sources of variation primarily reside in the inherent molecular instability of the biologicals (e.g., antibodies), appropriateness of sensing surfaces (immobilized capture molecules) and finally in the sensing principle and the corresponding instrumentation. In view of the bioassay features listed above, approaches are required where such variations can be autonomously compensated for. Different biosensing principles can be envisaged for detecting pesticides in water. Within this work, label-free assays were utilized for pesticide detection and quantitation. The biosensing surface plays a crucial role and the surface functionalization technology of CSEM can offer a variety of advantageous and competitive aspects. A special method designed is to couple target molecules to a dextran polymer (AtraDex) and covalently bind these polymers to OptoDex ${ }^{\mathrm{TM}}$ surfaces. The advantages are: (i) robust immobilization via multiple photo-bonding sites; (ii) well designable surface properties; and (iii) suppression of non-specific binding due to the dextran basis of both, capture and cross-linker (OptoDex $\left.{ }^{\mathrm{TM}}\right)$ molecules. In addition, AtraDex surfaces are very 
stable in ambient environment and re-generable. The synthesis of small molecules to dextran polymers seems therefore the method of choice also for other pesticides (or drugs) and water contaminants.

\section{Materials and Methods}

\subsection{Reagents}

All chemicals, unless specified, were obtained from Sigma-Aldrich (Switzerland). Mouse IgG and goat-anti mouse IgG antibodies were purchased from Thermo-Fisher (Switzerland). Gold nanoparticles of $40 \mathrm{~nm}$ diameter was purchased from Metalor (Switzerland), the conjugate of gold nanoparticle and goat anti-mouse IgG antibody was prepared in house. Atrazine was obtained from Labor Dr. Ehrenstorfer, Augsburg. Aqueous solutions of atrazine were prepared and final concentrations were quantitated optically $\left(\varepsilon_{263}=35^{\prime} 000 \mathrm{M}^{-1} \cdot \mathrm{cm}^{-1}\right.$ [1]). Monoclonal anti-atrazine antibodies were purchased either from Enzo Life Sciences, Switzerland (monoclonal anti-atrazine antibody [1F9]), or from Abcam ${ }^{\mathrm{TM}}$, Cambridge UK (monoclonal anti-atrazine antibody [8.F.20]). Concentrated stock solutions of antibodies were kept at $4{ }^{\circ} \mathrm{C}$ (Enzo) or at $-20{ }^{\circ} \mathrm{C}$ (Abcam). Prior to use, the antibodies were diluted with phosphate buffered saline pH 7.4 (PBS). Atrazine multivalent antigen-carrier dextran conjugate (AtraDex) was synthesized in house. The photo-crosslinkable polymer $\left(\mathrm{OptoDex}^{\circledR}\right)$ used to functionalize the chips and related technologies were developed by CSEM (Switzerland).

\subsection{Waveguide Grating Biosensor}

Immunoassays for the detection of atrazine were performed with the so-called wavelengthinterrogated optical sensing platform (WIOS) developed at CSEM. Details of the instrumentation have been given elsewhere [2]. Briefly, the detection of WIOS is based on changes of effective refractive index of a waveguide grating upon adsorption of biomolecules. The waveguide chips for WIOS consist of a thin $\mathrm{Ta}_{2} \mathrm{O}_{5}$ film deposited on a nano-structured glass substrate (Figure 1). The sensitivity of the instrument was shown to be below $10^{-6}$ refractive index units for bulk refractometry and the limit of detection for the adsorption of small molecules corresponded to a surface coverage of $0.3 \mathrm{pg} / \mathrm{mm}^{2}$. This feature makes it possible to analyze several pesticides with the different pads and some as reference for the correction of analytic matrix variations.

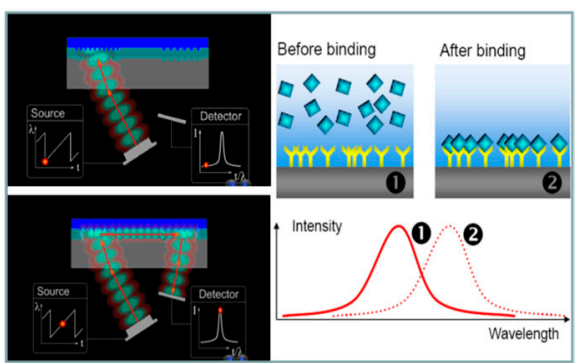

(a)
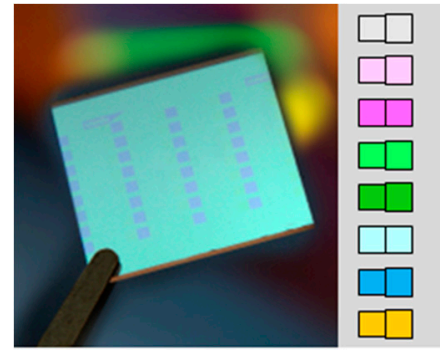

(b)

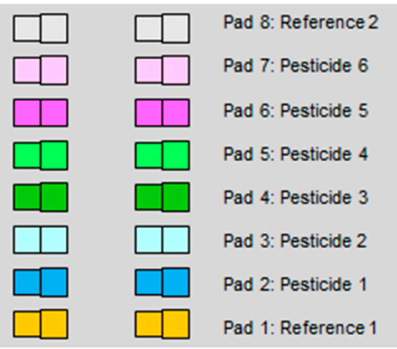

Pad 1: Reference 1

Figure 1. WIOS chip design and layout. (a) optical scheme showing the incoming tunable light from the VCSEL, the detection photodiode, and the structure of the waveguide chip with the gratings; a flow cell is mounted on top of the waveguide and allows flowing the liquid sample; (b) waveguide chip with the flow path of the liquid over the eight measurement pads, and sensing chip surface for the detection of up to 6 pesticides in parallel.

\subsection{Chip Functionalization}

A dextran-based atrazine multivalent antigen-carrier conjugate (AtraDex) was synthesized from amino dextran and carboxy-atrazine (6-(Aza-6-cloro-4-isopropylamino-pyrimidin-2yl)-amino) hexanoic acid): custom synthesized according to Fatibello et al. [3], applying EDC/NHS coupling chemistries. Multivalent atrazine carrier AtraDex was immobilized on the waveguide input gratings using the OptoDex ${ }^{\circledR}$ technology developed by CSEM. OptoDex ${ }^{\circledR}$ is a dextran-based photo- 
crosslinkable polymer that covalently binds biomolecules to surfaces upon UV exposure. The reagents are spotted onto any of the eight measurement pads using a NanoPlotterTM dispensing system (GeSim, Germany). In addition to the excellent stability of the bound molecules the polymer layer provides passivation, preventing the non-specific adsorption of biomolecules. In each WIOS chip, four measurement pads were functionalized with an optimized amount of AtraDex and four others were used as reference channels.

\section{Results}

\subsection{Competitive Immunoassay for Detection of Atrazine}

The assay format is a competitive one, where analogues of the molecules to be detected (in the present case analogues of atrazine) are immobilized on the surface (Figure 2). The competitive assay offers the advantage that the immobilized target molecules are usually more robust than antibodies. This is an essential prerequisite for surface regeneration and long shelf-life of the biosensor.
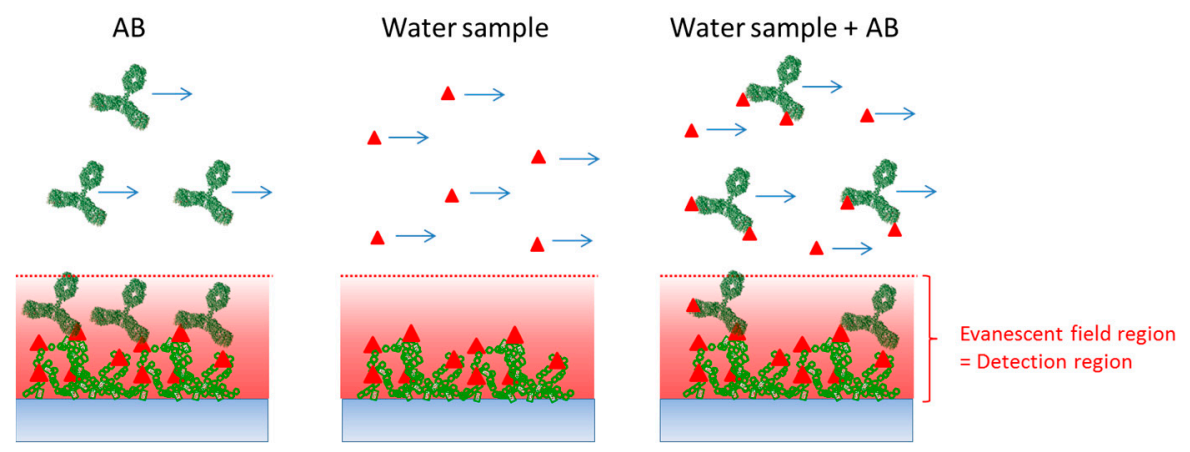

Figure 2. Schematic of the competitive bioassay principle used for the detection of atrazine.

An advantage of the system over conventional detection systems is to be able to measure eight channels simultaneously. This feature makes it possible to analyze several contaminants in the same water sample. An example of atrazine calibration curves obtained by signal amplification using goldlabeled secondary antibody can be seen in Figure 3. Standard curves were fitted to dose-response data by using a 4-parameter model. As the assays conducted with the WIOS label-free was always of the competitive type, standard curves have their maximum at zero concentration and their minimum at infinite concentrations. The detection limit is reached at $0.05 \mu \mathrm{g} / \mathrm{L}$ of free atrazine.

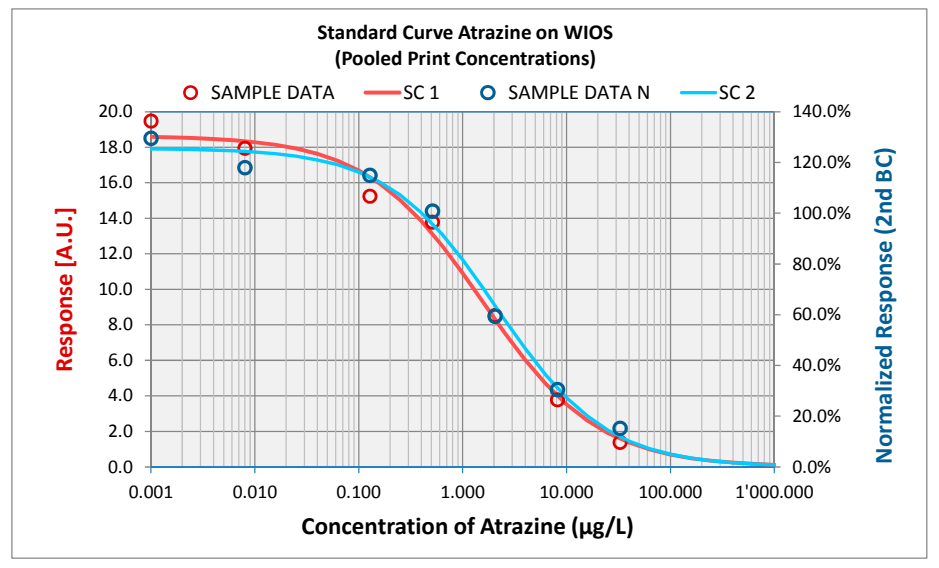

Figure 3. Fitting to sample data with an atrazine calibration series. The red fit curve corresponds to pad pooled response values without built-in calibration (in Arbitrary WIOS Units), the blue curve to calibrated data (in \%, right $y$-axis) using the second response curve in the assay (see above). Note that in the following percentage values always correspond to normalized responses using the built-in calibration. 


\subsection{Regenerability and Stability of Sensor Surface}

Regenerability of analytical chips for biodetection in general, and for contaminants in water in particular, is a challenge with considerable commercial consequences. Optical chips for label-free detection attain reasonable cost efficiency when both multiplexing and regenerability can be considered. It is therefore essential to provide appropriately functionalized surfaces which are covalently bonded, immunologically competent and regenerable. OptoDex ${ }^{\mathrm{TM}}$ based surface engineering is additionally beneficial due to its property of suppressing non-specific binding. The later point is of major importance regarding contaminant analysis in water. To date, the investigations indicate that surfaces with photo-bonded AtraDex can be regenerated more than 80 times, stored in buffer at least for 2 months and in dry state for at least one year without losing their properties. The quality of generated data has been significantly improved with inclusion of the built-in calibration.

\subsection{Matrix Effects on the Atrazine Assay}

Further piloting experiments concerned investigations of matrix and interfering effects. Interferents are assay-disturbing components which may arise in real samples. Performed investigations are dual aimed. For bioanalytics it is essential to know which matrix may possibly interfere with the assay and thus falsify the result. Moreover, is it possible to identify interfering effects and correct the primary result accordingly? Matrix effects have been studied so far with tap water and lake water (Lake of Neuchatel, Switzerland). The matrix component in these assays accounts for $94 \%$ by volume. The results are summarized in Table 1 . As compared to deionized water, neither tap water nor lake water interfere with the atrazine detection assay.

Table 1. Matrix effects on atrazine detection.

\begin{tabular}{ccccccc}
\hline Atrazine & $\mathbf{0 . 0 0 1} \boldsymbol{\mu g} / \mathrm{L}$ & $\mathbf{0 . 5 1 2} \boldsymbol{\mu g} / \mathbf{L}$ & $\mathbf{2 . 0 5} \boldsymbol{\mu g} / \mathbf{L}$ \\
\hline Signal/Recov. Atrazine & Calibration $\mathbf{( \% )}$ & $\boldsymbol{\mu g} / \mathrm{L}$ & Calibration $\mathbf{( \% )}$ & $\boldsymbol{\mu g} / \mathrm{L}$ & Calibration (\%) & $\boldsymbol{\mu g} / \mathbf{L}$ \\
\hline Assay buffer & 129 & 0.000 & 106 & 0.380 & 64 & 2.42 \\
Tap water & 134 & 0.000 & 93 & 0.760 & 68 & 2.07 \\
Lake water & 123 & 0.026 & 97 & 0.720 & 69 & 1.92 \\
\hline
\end{tabular}

\section{Conclusions}

In summary, we can offer a system for online monitoring of water contaminants with the following properties: (a) Competitive immunoassay with antigen-carrier (AtraDex) on surface; (b) Label-free sensing for real-time measurement and reaction kinetics; (c) Multiple sensing surfaces with different characteristics; (d) In-assay calibration/normalization; (e) Regenerable biochip more than 80 times; (f) Detection limit of atrazine achieved at $0.05 \mu \mathrm{g} / \mathrm{L} ;(\mathrm{g})$ Computational bioanalytics; (h) Compensation of interfering effects.

Conflicts of Interest: The authors declare no conflict of interest.

\section{References}

1. Azenha, M.E.D.G.; Burrows, H.D.; L, M.C.; Coimbra, R.; Ândez, M.I.F.; GarcõÂa, M.V.; Peiteado, M.A.; Santaballa, J.A. Kinetic and mechanistic aspects of the direct photodegradation of atrazine, atraton, ametryn and 2-hydroxyatrazine by $254 \mathrm{~nm}$ light in aqueous solution. J. Phys. Org. Chem. 2003, 16, 498-503.

2. Cottier, K.; Wiki, M.; Voirin, G.; Gao, H.; Kunz, R.E. Label-free highly sensitive detection of (small) molecules by wavelength interrogation of integrated optical chips. Sens. Actuators B Chem. 2003, 91, 241-251.

3. Fatibello, F.O.; Souza, M.G.; Vieira, I. Flow injection spectrophotometric determination of phenolic compounds in wastewaters using peroxidase of zucchini (Cucurbita pepo). Ecletica Quím. 2002, 27, 51-66. 